\title{
Electronic and magnetic behaviors of calcined material from a neodymium-O- phenylene-O-ytterbium hybrid copolymer
}

Hideo Matsui*, Hirokazu Miyazaki*, Keigo Otsuki*, Emi Kunimitsu*, Yasuhiro Seguchi*, Tetsuro Kawahara** and Masakuni Yoshihara*,****

A networked neodymium-O-phenylene-O-ytterbium ternary hybrid copolymer was calcined under vacuum at 400,500 , 600 , and $700{ }^{\circ} \mathrm{C}$ to obtain black-colored materials composed of carbon clusters and nano-sized $\mathrm{Yb}_{2} \mathrm{O}_{3}$ and $\mathrm{Nd}$ compound particles. ESR spectral examinations showed that the calcined materials have a free electron on carbon clusters via electron transfer between carbon clusters and metal compounds. From the changes of ESR signal intensity of the calcined materials under magnetic field impression, spin relaxation in the neodymium atom is presumed to be controlled by a spin interaction with the ytterbium atom.

KEYWORDS : $\mathrm{Nd}$ compound, $\mathrm{Yb}_{2} \mathrm{O}_{3}$, Electron transfer, Magnetic field-response characteristic

\section{Introduction}

Hybrid materials composed of organic and inorganic units have been attracting much attention, because the properties of the materials are expected to be controlled by changing the natures and/or ratios of organic and inorganic components ${ }^{1)}$, 2). Many studies focused on metal-organic coordination polymerization have been reported for building up multi-dimensional networks ${ }^{3)-7)}$. We have considered that alternating organic-metal hybrid copolymers with covalent bonding can constitute new types of multi-aggregated composite materials ${ }^{8)-12)}$, which are expected to exhibit unique electronic, magnetic, and optical properties.

We have recently reported the synthesis of a ternary neodymiumO-phenylene-O-ytterbium hybrid copolymer I (Scheme 1), in which an electron transfer between the phenylene moiety and the ytterbium atom through the neodymium atom takes place to form a

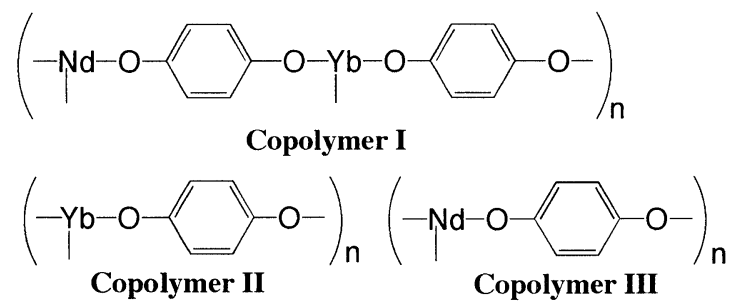

Scheme 1 Syntheses of copolymers I, II, and III. stable free electron on the phenylene moiety ${ }^{13)}$. Here, we consider that the calcination of copolymer I under reducing atmosphere will give a new type of a composite material composed of carbon clusters and each metal oxide, possibly neodymium oxide and ytterbium oxide, and such a material will show unique electronic, magnetic, and/or photochemical natures. In the present study, we describe the electronic and magnetic behaviors of the material obtained by calcinating copolymer I under vacuum.

\section{Experimental}

\subsection{Reagents}

Commercially available neodymium chloride, ytterbium chloride, 1,4-hydroquinone, and 1,8-bis (dimethylamino) naphthalene (proton sponge) were used. Other reagents and solvents were purified by standard procedures.

\subsection{Syntheses of copolymers I, II, and III}

Copolymers I, II, and III (Scheme 1) were synthesized according to the previously reported procedures ${ }^{11)}{ }^{13)}$.

Copolymer I was synthesized by the first reaction of 1,4hydroquinone $(3.0 \mathrm{mmol}, 0.34 \mathrm{~g})$ with neodymium chloride $(1.0 \mathrm{mmol}, 0.25 \mathrm{~g})$ at $-70{ }^{\circ} \mathrm{C}$ followed by the second reaction with ytterbium chloride $(1.0 \mathrm{mmol}, 0.28 \mathrm{~g})$ at $-30^{\circ} \mathrm{C}$ in the presence of proton sponge $(6.0 \mathrm{mmol}, 1.28 \mathrm{~g})$. Copolymers II and III were synthesized by the reaction of 1,4-hydroquinone $(1.5 \mathrm{mmol}, 0.17 \mathrm{~g}$ ) with neodymium chloride $(1.0 \mathrm{mmol}, 0.25 \mathrm{~g})$ or ytterbium chloride 
$(1.0 \mathrm{mmol}, 0.28 \mathrm{~g})$, respectively, at room temperature in the presence of proton sponge $(3.0 \mathrm{mmol}, 0.64 \mathrm{~g})$.

\subsection{Calcination of copolymers}

$0.2 \mathrm{~g}$ of copolymer I in a porcelain crucible was calcined under vacuum with a heating rate of $5^{\circ} \mathrm{C} / \mathrm{min}$ and kept for $1 \mathrm{hr}$ at 400 , 500,600 , and $700{ }^{\circ} \mathrm{C}$, respectively, using an electric furnace, Denken KDF-75. Copolymers II and III were calcined by the same procedure at $500{ }^{\circ} \mathrm{C}$.

\subsection{Measurements}

TG-DTA analysis of copolymer I $(1.0 \mathrm{mg})$ was performed in nitrogen atmosphere using Rigaku TG-DTA-MS 8010. C and $\mathrm{H}$ contents were analyzed by coulometric titration using Yanaco CHN corder MT-6. Nd and $\mathrm{Yb}$ contents were analyzed using Shimadzu ICP-7500. X-ray diffraction (XRD) spectra were measured using Rigaku Mini Flex with $\mathrm{Cu}-\mathrm{K} \alpha$ source $(30 \mathrm{kV}, 15 \mathrm{~mA})$. Transmission electron microscopy (TEM) observations were done using Jeol JEM-3010 microscope (accelerating voltage : $300 \mathrm{kV}$ ). UV-VIS spectra were measured using Hitachi U-4000. Electron spin resonance (ESR) spectra were taken using Jeol JES-TE 200 spectrometer (sample weight : $5 \mathrm{mg}$, c. field ; $337.00 \mathrm{mT}$, power ; $1.00 \mathrm{~mW}$, sweep width ; $\pm 5 \mathrm{mT}$, modulation width ; $0.5 \mathrm{mT}$, time constant ; $0.1 \mathrm{sec}$, temp. ; room temp.). X-ray photoelectron spectra (XPS) measurements were carried out using Shimadzu ESCA-850 spectrometer.

\section{Results and discussion}

TG-DTA measurement of copolymer I under a nitrogen atmosphere with a heating rate of $5^{\circ} \mathrm{C} / \mathrm{min}$ (Fig.1) showed weight decrease with an endothermic reaction of $25 \sim 150{ }^{\circ} \mathrm{C}$ due to evaporation of volatile components and an exothermic reaction of $300 \sim 700{ }^{\circ} \mathrm{C}$ due to possibly carbonization of copolymer.

Copolymer I was calcined under vacuum with a heating rate of $5^{\circ} \mathrm{C} / \mathrm{min}$ and kept for 1 hour at $400,500,600$, and $700{ }^{\circ} \mathrm{C}$, respectively, to obtain calcined materials Ic-400, Ic-500, Ic-600, and Ic-700, respectively.

In order to check the calcination process of copolymer I and to determine the compositions of the materials, ICP and elemental analyses were performed (Table 1). The observed hydrogen contents in the calcined materials decreased with increasing calcination temperature, indicating that the carbonization of copolymer I proceeded. The molar ratios of neodymium to ytterbium contents in the calcined materials were in the range of $0.84 \sim 0.87$ (Table 1). XRD pattern of the Ic-700 showed clear peaks due to ytterbium oxide $\left(\mathrm{Yb}_{2} \mathrm{O}_{3}\right)$ at $2 \theta=29.3^{\circ}, 34.3^{\circ}, 49.4^{\circ}$, and $58.6^{\circ}$, and those of Ic-400, Ic-500, Ic-600 showed broad peak at $29.3^{\circ}$ (Fig.2). On the other hand, although the ICP analyses showed the presence of $\mathrm{Nd}$ atom (Table 1), clear peak due to elemental neodymium or any of its compounds could not be observed. It can be supposed

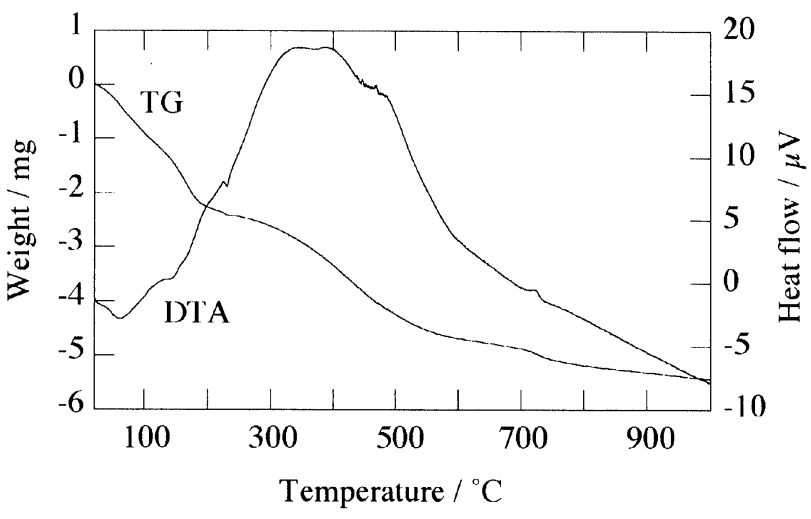

Fig.1 TG-DTA analysis of copolymer I.

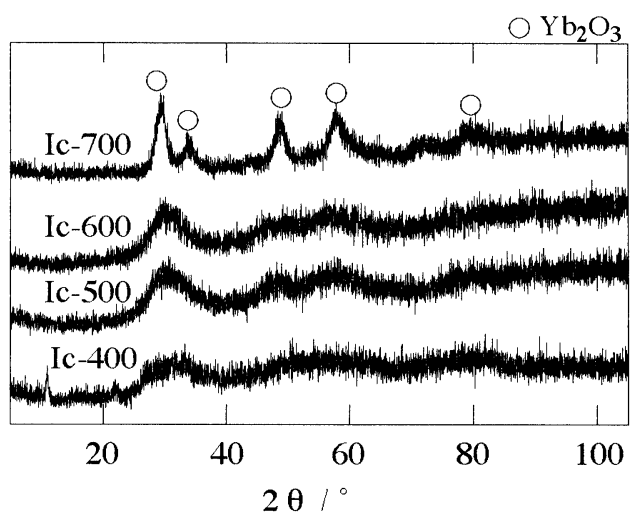

Fig.2 X-ray diffraction patterns of calcined materials Ic.

Table 1 ICP and elemental analyses of copolymer and calcined materials.

\begin{tabular}{|c|c|c|c|c|c|}
\hline \multirow{2}{*}{ Material } & \multicolumn{4}{|c|}{ Found / \% } & \multirow{2}{*}{$\begin{array}{l}\text { Molar ratio } \\
\text { of } \mathrm{Nd} / \mathrm{Yb}\end{array}$} \\
\hline & $\mathrm{Nd}$ & $\mathrm{Yb}$ & $\mathrm{C}$ & $\mathrm{H}$ & \\
\hline I & 18.60 & 20.49 & 13.77 & 2.82 & 1.09 \\
\hline Ic-400 & 19.80 & 27.46 & 14.63 & 2.31 & 0.87 \\
\hline Ic-500 & 21.07 & 29.01 & 12.26 & 1.31 & 0.87 \\
\hline Ic- 600 & 23.30 & 33.29 & 11.23 & 1.01 & 0.84 \\
\hline Ic-700 & 26.11 & 36.11 & 10.16 & 0.67 & 0.87 \\
\hline
\end{tabular}

that neodymium exist as oxide $\left(\mathrm{Nd}_{2} \mathrm{O}_{3}\right)$. TEM images (Fig.3) showed the presence of ultra-fine particles with diameters of $1 \sim 5$ $\mathrm{nm}$, possibly ytterbium oxide and neodymium compound, in the matrix of amorphous carbon clusters with diameters of $100 \sim 500$ $\mathrm{nm}$. Thus, the calcined materials are considered to be composed of carbon clusters and nano-sized ytterbium oxide $\left(\mathrm{Yb}_{2} \mathrm{O}_{3}\right)$ and neodymium compound particles.

The electronic behaviors of the calcined materials were examined. UV-VIS spectral measurements of copolymer I and calcined materials Ic's showed absorption bands with high intensities over the entire range of wavelength (Fig.4), indicating that the electron transfer excitation takes place easily. In order to verify these findings, ESR spectra of I and Ic's were measured to show the signal due to a free electron at $337 \mathrm{mT}(\mathrm{g}=2.003)($ Fig.5 $)$. The radical spin quantities $(r s q)$ of the materials were determined by a double 


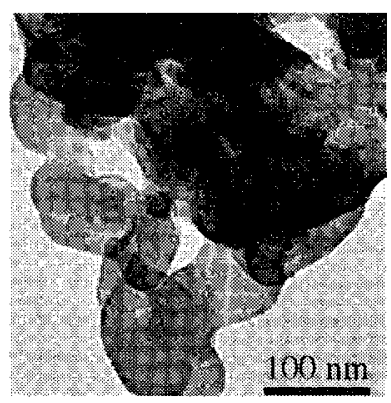

$\mathrm{Ic}-400$

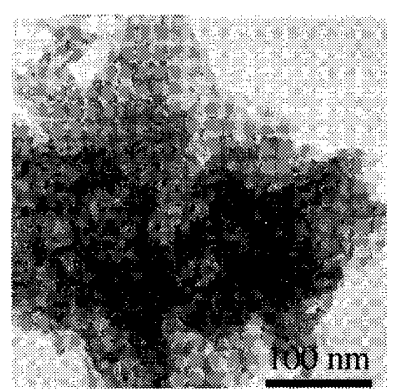

Ic- 600

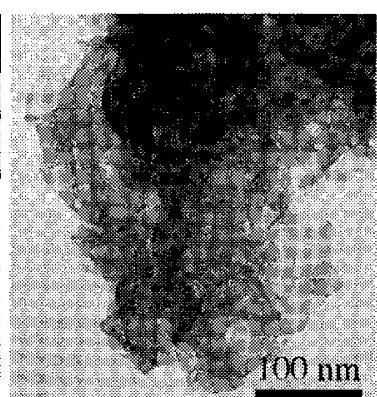

Ic -500

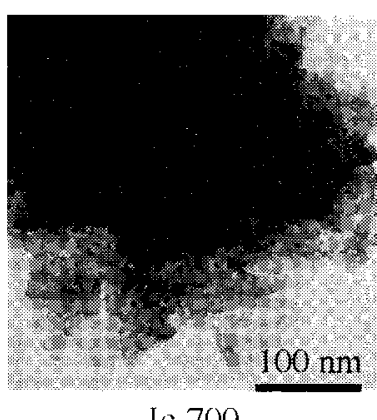

$\mathrm{Ic}-700$
Fig.3 TEM images of calcined materials Ic.

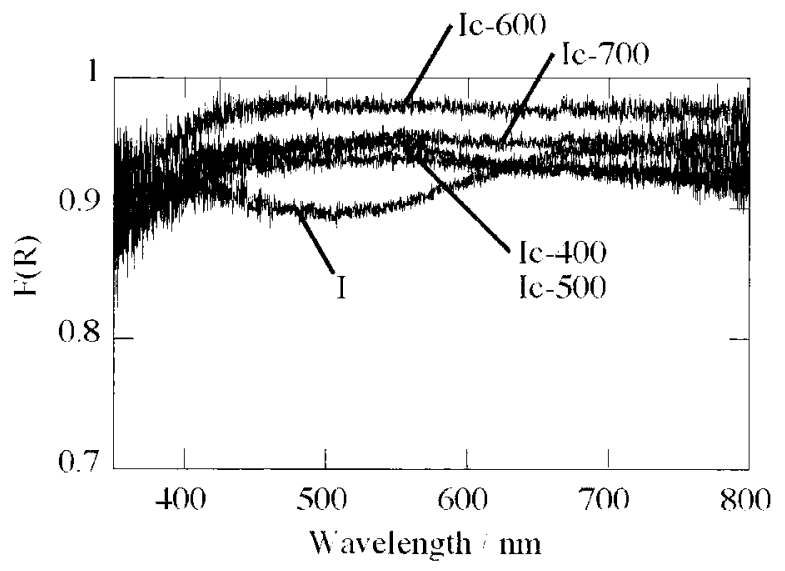

Fig.4 UV-VIS spectra of copolymer I and calcined materials Ic.

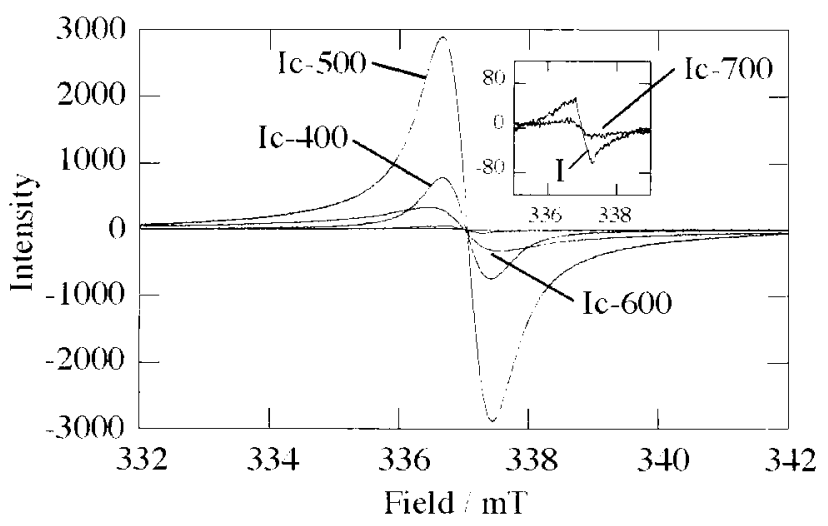

Fig.5 ESR spectra of copolymer I and calcined materials Ic.

integrating calculation of the differential absorption line with the use of DPPH (Table 2). The rsq values were larger in the order of Ic $-500>$ Ic $-600>$ Ic $-400>$ Ic-700 $>$ copolymer I, and the $r s q$
Table 2 Radical spin quantities ( $r s q$ ) of copolymer and calcined materials.

\begin{tabular}{ccc}
\hline Material & Radical spin quantity $(\mathrm{rsq}) / \mathrm{spins}^{\cdot \mathrm{g}^{-1}}$ & Ratio(Ic/I $)$ \\
\hline I & $5.62 \times 10^{18}$ & 1 \\
Ic-400 & $8.32 \times 10^{19}$ & 15 \\
Ic-500 & $5.25 \times 10^{20}$ & 93 \\
Ic-600 & $1.77 \times 10^{20}$ & 31 \\
Ic-700 & $1.32 \times 10^{\mathrm{i9}}$ & 2 \\
\hline
\end{tabular}

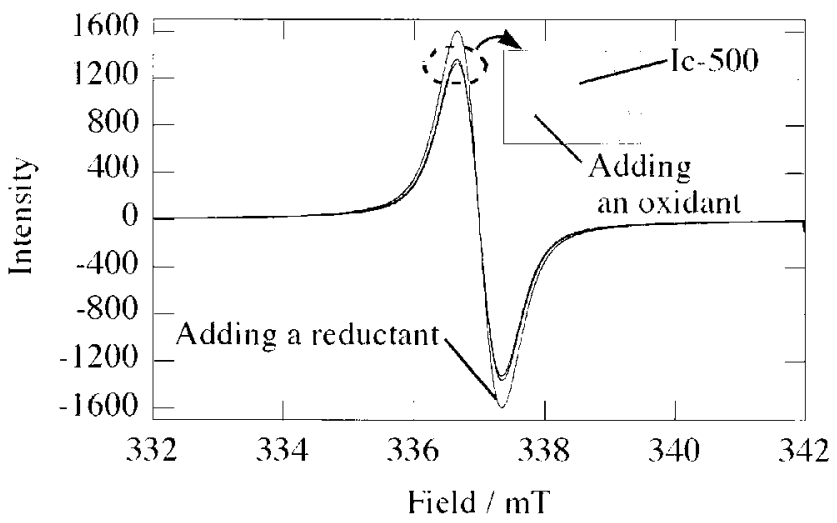

Fig.6 ESR spectra of $500^{\circ} \mathrm{C}$-calcined material (Ic-500) in the presence of reductant or oxidant.

value of Ic-500 was about 93 times as large as that of copolymer I. Our opinion is that the electron transfer between carbon clusters and metal oxides takes place to form a stable radical on carbon clusters, and that although the calcination treatment is indispensable for the electron transfer, there exist a optimal structure or degree of crystallization which could be obtained by calcination at ca. $500^{\circ} \mathrm{C}$ with the condition of this study. As shown in Fig.6, the addition of an oxidant (benzoquinone) to Ic-500 decreased slightly the signal intensity at $337 \mathrm{mT}$, but the signal intensity increased with the addition of a reductant (pyrogallol), suggesting that the signal is due to an anion radical formed on the carbon clusters. In other words, an electron transfer from the metal oxide to the carbon clusters may take place. In order to examine the electron transfer feature in Ic-500, individual binary hybrid copolymers, i.e. phenylene-Oytterbium hybrid copolymer II and phenylene-O-neodymium hybrid copolymer III (Scheme 1), were synthesized, and the copolymers were calcined at $500{ }^{\circ} \mathrm{C}$ under vacuum for $\mathrm{l} \mathrm{hr}$ to obtain calcined materials IIc-500 and IIlc-500, respectively (Table 3).

Table 4 summarizes the results of XPS analyses of calcined materials and metal oxide. The values of $4 \mathrm{~d} 5 / 2$ of $\mathrm{Nd}$ in the calcined materials Ic and IIIc-500 were considerably higher than that in $\mathrm{Nd}_{2} \mathrm{O}_{3}$, and those of $4 \mathrm{~d} 5 / 2$ of $\mathrm{Yb}$ in the calcined materials were slightly higher than that in $\mathrm{Yb}_{2} \mathrm{O}_{3}$, suggesting that the electron density of $\mathrm{Nd}$ atom in the calcined materials decreased in comparison with that of $\mathrm{Yb}$ atom. Therefore, an electron transfer in Ic-500 is considered to take place in the process of $\mathrm{Nd}$ compound $\rightarrow$ carbon clusters $\rightarrow \mathrm{Yb}_{2} \mathrm{O}_{3}$ 
Table 3 Elemental analyses of copolymers II and III and calcined materials IIc-500 and IIIc-500.

Table 4 Binding energies $(\mathrm{eV})$ of metal oxides and calcined materials.
Fig.8 ESR spectra of $500 \mathrm{t}$-calcined material (IIc-500) under

magnetic field impression
Scheme 2 A proposed electron transfer process.

Fig.7 ESR spectra of 500 t-calcined material (Ic-500) under magnetic field impression.

-car bon cl ust er s( Schene 2).

Both ytterbium and neodymium atoms have an odd electron in

the $4 \mathrm{f}$ orbital, and thus, the calcined material is expected to show a

unique magnetic characteristic. As shown in Fig.7, the impression

of strong magnetic field (magnetic field strength ; 5 G) to Ic-500

increased the ESR signal intensity, which, after eliminating

magnetic-field, gradually increased, then decreased, and finally
Fig.9 ESR spectra of $500 \mathrm{t}$-calcined material (IIIc-500) under magnetic field impression.

returned to the original intensity, indicating that hysteresis phenomena appeared in the demagnetization process of Ic-500 ESR spectra of IIc-500 and IIIc-500 under magnetic field impression (Figs.8 and 9) showed hysteresis phenomena similar to Ic-500. From the results given in Figs.7, 8 and 9, the decrease in signal intensity during $24 \mathrm{~h}$ after eliminating magnetic field was relatively larger for IIIc-500 (Nd system) and smaller for IIc-500 (Yb system). It suggests that, in Ic-500 (Nd-Yb system), a rather fast spin relaxation in the neodymium atom may be controlled by the ytterbium atom ; thus, a spin interaction between ytterbium and neodymium atoms may take place. In other words, a spin transport in Ic-500 is considered to be brought by an electron transfer between neodymium and ytterbium atoms. It should be noted that the signal intensities in Ic-500 and IIIc-500 at $24 \mathrm{~h}$ after eliminating magnetic field were lower than those before magnetic field application, and its reason is not yet cleared.

We have found that the neodymium compound-ytterbium oxidecarbon cluster composite material has a magnetic field-response characteristic. We believe that similar functions can be achieved by the combination of carbon clusters and semiconductors having an odd electron in f-orbital of metals, and the materials will be useful as magnetic devices. 


\section{References}

1) J. Cho and K. Sul, Polymer 42 (2001) 727.

2) Z. Huang and K. Qiu, Polymer 38 (1997) 521.

3) H. Bonneman and N. Waldofner, Chem. Mater. 14 (2002) 1115.

4) D. M. Young, U. Geiser, A. J. Schultz and Z. Wang, J. Am. Chem. Soc. 120 (1998) 1331.

5) B. F. Hoskins, R. Robson and D. A. Slizys, J. Am. Chem. Soc. 119 (1997) 2952.

6) D. Hagrman and J. Zubieta, J. Chem. Soc. Chem. Commun. 1998 2005-2006.

7) C. J. Kepert and M. J. Rosseinsky, J. Chem. Soc. Chem. Commun. $199831-32$
8) H. Matsui, A. Okada and M. Yoshihara, J. Mater. Sci. Lett. 20 (2001) 1151-1153.

9) M. Yoshihara, H. Oie, A. Okada, H. Matsui and S. Ohshiro, Macromolecules 35 (2002) 2435-2436.

10) H. Matsui, A. Okada, T. Takeuchi and M. Yoshihara, Electrochemistry 11 (2002) 842.

11) M. Yoshihara, T. Shino, K. Otsuki, H. Matsui and S. Ohshiro, J. Mater. Sci. Lett. 21 (2002) 1051-1053.

12) H. Matsui, K. Otsuki and M. Yoshihara, J. Macr. Sci. Part A-Appl. Chem. 42 (2005) 633.

13) H. Matsui, K. Otsuki and M. Yoshihara, J. Jpn. Soc. Colour Mater. 78 (2005) 122 (in Japanese). 